\section{(C) OPEN ACCESS}

\title{
Epidemiology of valvular heart disease in a Swedish nationwide hospital-based register study
}

\author{
Pontus Andell, ${ }^{1}$ Xinjun Li, ${ }^{2}$ Andreas Martinsson, ${ }^{1}$ Charlotte Andersson, ${ }_{1}^{3}$ \\ Martin Stagmo, ${ }^{1}$ Bengt Zöller, ${ }^{2}$ Kristina Sundquist, ${ }^{2}$ J Gustav Smith ${ }^{1,4}$
}

\begin{abstract}
- Additional material is published online only. To view please visit the journal online (http://dx.doi.org/10.1136/ heartjnl-2016-310894).

${ }^{1}$ Department of Cardiology, Clinical Sciences, Lund University and Skåne University Hospital, Lund, Sweden ${ }^{2}$ Center for Primary Health Care Research, Lund University, Malmö, Sweden

${ }^{3}$ Department of Cardiology, Copenhagen University Hospital Gentofte, Hellerup, Denmark ${ }^{4}$ Program in Medical and Population Genetics, Broad Institute of Harvard and Massachusetts Institute of Technology, Cambridge, Massachusetts, USA
\end{abstract}

\section{Correspondence to} Dr Pontus Andell, Department of Cardiology, Lund University, SE22185 , Lund, Sweden; pontus. andell@gmail.com

Received 11 November 2016 Revised 14 March 2017 Accepted 16 March 2017 Published Online First 21 April 2017

\section{Linked}

- http://dx.doi.org/10.1136/ heartjnl-2017-311472

\section{CrossMark}

To cite: Andell P, Li X Martinsson $A$, et al. Heart 2017:103:1696-1703.

\section{ABSTRACT}

Objective Transitions in the spectrum of valvular heart diseases (VHDs) in developed countries over the 20th century have been reported from clinical case series, but large, contemporary population-based studies are lacking.

Methods We used nationwide registers to identify all patients with a first diagnosis of VHD at Swedish hospitals between 2003 and 2010. Age-stratified and sex-stratified incidence of each VHD and adjusted comorbidity profiles were assessed.

Results In the Swedish population ( $n=10164$ 211), the incidence of VHD was 63.9 per 100000 person-years, with aortic stenosis (AS; 47.2\%), mitral regurgitation ( $\mathrm{MR} ; 24.2 \%$ ) and aortic regurgitation (AR; 18.0\%) contributing most of the VHD diagnoses. The majority of VHDs were diagnosed in the elderly (68.9\% in subjects aged $\geq 65$ years), but pulmonary valve disease incidence peaked in newborns. Incidences of $A R, A S$ and $M R$ were higher in men who were also more frequently diagnosed at an earlier age. Mitral stenosis (MS) incidence was higher in women. Rheumatic fever was rare. Half of AS cases had concomitant atherosclerotic vascular disease (48.4\%), whereas concomitant heart failure and atrial fibrillation were common in mitral valve disease and tricuspid regurgitation. Other common comorbidities were thoracic aortic aneurysms in AR (10.3\%), autoimmune disorders in MS (24.5\%) and abdominal hernias or prolapse in MR (10.7\%) and TR (10.3\%).

Conclusions Clinically diagnosed VHD was primarily a disease of the elderly. Rheumatic fever was rare in Sweden, but specific VHDs showed a range of different comorbidity profiles. Pronounced sex-specific patterns were observed for AR and MS, for which the mechanisms remain incompletely understood.

\section{INTRODUCTION}

A hundred years ago, valvular heart disease (VHD) was considered the major heart disease and a leading cause of death. ${ }^{1}$ In particular, rheumatic heart disease, typically presenting with mitral stenosis (MS) and mitral regurgitation (MR), was endemic and spurred both the development of open heart surgery and echocardiography. ${ }^{2}$ Over the 20th century, the heart disease spectrum transitioned in western countries to predominance of coronary artery disease, and the incidence of rheumatic heart disease declined, although rheumatic heart disease remains common in sub-Saharan Africa, south-central Asia and the Pacific. ${ }^{45}$
In the 21st century, VHD has increasingly become recognised as a manifestation of degenerative processes related to ageing. ${ }^{6}$ With decreasing incidence and mortality of coronary artery disease ${ }^{8}$ and longer lifespan, the burden of VHD on health systems is expected to increase. ${ }^{9}{ }^{10}$ Fortunately, in parallel, technical and surgical advances have led to increasing availability of surgical treatment in many elderly patients who were previously deemed unsuitable candidates, resulting in improved survival and quality of life. ${ }^{11-14}$ In addition, endovascular therapies have emerged and evolved rapidly and are now available for all four heart valves. ${ }^{15-20}$ Initial clinical trials have also suggested that pharmacological inhibition of neurohormonal pathways may slow detrimental myocardial remodelling processes in VHD. ${ }^{21} 22$

Contemporary epidemiological information on VHD is important to better understand this changing disease spectrum and allocate healthcare resources accordingly. Currently, most epidemiological knowledge of VHD on the population level has been extrapolated from cross-sectional studies in the USA, which have been underpowered for analysis of individual valve diseases. ${ }^{73} \mathrm{We}$, therefore, aimed to use the unique nationwide registers available in Sweden to explore the contemporary epidemiological characteristics across the whole spectrum of VHDs.

\section{METHODS}

\section{Nationwide registers}

The entire Swedish population between 2003 and 2010 was included in the study using nationwide registers linked to 141 healthcare providers, including all hospitals and specialised outpatient clinics, both public and private. All patients with a first primary or secondary diagnosis of VHD were identified from the National Patient Register (NPR) for any of the eight VHDs: aortic stenosis (AS), aortic regurgitation (AR), mitral stenosis (MS), mitral regurgitation (MR), pulmonary stenosis (PS), pulmonary regurgitation (PR), tricuspid stenosis (TS) and tricuspid regurgitation (TR). Diagnoses of comorbid conditions were also identified from the NPR. The NPR includes International Classification of Diseases (ICD) diagnosis codes from all hospital inpatient and outpatient visits in Sweden. Reporting to the NPR is mandatory, and departmental reimbursements from the Swedish tax-financed healthcare system are based on the diagnosis codes. We protected anonymity by replacing the personal identification number with a 
serial number when linking data. The ethics committee at Lund University approved the study.

\section{Diagnostic definitions}

Diseases diagnosed and recorded in the NPR were based on ICD definitions from the WHO. In Sweden, the 10th version of ICD was used from 1997 and onwards. All VHD diagnoses in this study were, thus, classified according to ICD-10. The ICD codes used for VHD diagnoses are shown in online supplementary table 1 . The validity of diagnoses in the NPR has previously been shown to be high (positive predictive value $>85 \%$ ) for many diagnoses, including AS. We have also previously shown AS diagnoses in the NPR to be based mainly on echocardiographic assessments and typically represent moderate to severe severity rating. ${ }^{24-26}$

For the remaining VHD diagnoses in the NPR, we conducted validation analyses using medical records from participants of the large population-based cohort Malmö Diet and Cancer Study. We estimated that we would have $87 \%$ power to detect a significant (at $\mathrm{p}<0.05)$ difference in validity of $10 \%$ (null hypothesis proportion set to 0.9 , true proportion set to 0.8 ) with 100 cases, a power of $56 \%$ with 50 cases and a power of only $37 \%$ with 20 cases. Due to very few individuals in the validation cohort study with a diagnosis of PS (four cases), PR (one case) and TS (five cases), these were excluded from the validation analyses. In summary, the validation analyses found generally high validities for all VHDs studied (positive predictive value $>85 \%$ ), and disease severity was moderate or severe in the majority of cases (online supplementary tables 3-6 and online supplementary figures 1-4).

Comorbid conditions in the NPR diagnosed between 2003 until 2010 were classified in 15 different comorbidity groups, representing major potential pathophysiological mechanisms (online supplementary table 2). For rheumatic fever and non-valvular congenital heart disease, which may be diagnosed long before onset of VHD, we also included ICD-9 and ICD-8 codes to extend the timeframe. In Sweden, the eighth version of ICD was used between 1968 and 1986, and the ninth version of ICD was used from 1987 until 1996.

\section{Statistical analyses}

All patients with any diagnosis of the eight VHDs between 2003 and 2010 were included in the analysis and related to the entire Swedish population during the same period. Sex-specific incidence rates per 100000 person-years were computed and stratified by 5 -year age intervals $(0-4,5-9,10-14$ up to $85+)$. Cases diagnosed with VHD in the preceding 3-year period (2000-2002) were excluded to allow estimation of incidence rates. CIs for incidence rates were estimated under the assumption of a Poisson distribution. Comorbidities were assessed up to the last available follow-up time point. Associations of VHDs with each of the 15 comorbidity groups were explored cross-sectionally using logistic regression models adjusted for age and sex and presented as ORs with 95\% CIs. Unadjusted comorbidity rates across VHDs were also calculated. Statistical analyses including power calculations (proc power) for the validity analyses were performed in SAS V.9.3 (SAS Institute Inc. Cary, NC, USA) and SPSS V.23.0 (IBM, New York, NY, USA). Figure 3 was created with GraphPad Prism V.6.0 (GraphPad Software, San Diego, California, USA).

\section{RESULTS}

\section{Population and stratified incidences}

The entire Swedish population was included in the study population resulting in 10164211 individuals during the study period. Incidence rates of VHDs in Sweden between 2003 and 2010 are shown in table 1 . The highest incidence in both men and women was observed for AS (37.8 and 24.2 per 100000 person-years) followed by MR (21.3 and 16.0 per 100000 person-years) and AR (19.7 and 10.8 per 100000 person-years). Collectively, these three valve diseases comprised the majority of VHDs in the Swedish population (89.4\% of all diagnosed cases). Age-stratified and sex-stratified incidence rates are shown in figure 1 for VHDs of the left-sided heart chambers and in figure 2 for VHDs of the right-sided heart chambers. The majority of VHDs were diagnosed in the elderly (68.9\% in subjects aged $\geq 65$ years). Diseases of the aortic and mitral valve as well as TR increased continuously with increasing age, starting from the fourth or fifth decade of life with peak incidences in the seventh to eighth decades. In contrast, incidence of pulmonary valve disease and the least common VHD, TS, peaked over the first decade of life. Marked sex-specific differences were observed for AR incidence, with a male preponderance across all age groups (figure 1). The diagnosis code commonly used for bicuspid aortic valves (Q23.1: congenital insufficiency of aortic valve) was twofold more common in men with aortic valve disease (AS and AR combined) as compared with women, contributing $4.3 \%$ and $2.4 \%$ of cases, respectively. AS and MR also had higher incidences in men but

Table 1 Study population, cases and incidence rates of valvular heart disease in Sweden between 2003 and 2010

\begin{tabular}{|c|c|c|c|c|c|c|c|c|c|c|}
\hline \multirow[b]{2}{*}{ Population } & \multicolumn{5}{|c|}{ Men ( $n=5060$ 355) } & \multicolumn{5}{|c|}{ Women $(n=5103856)$} \\
\hline & $\mathrm{N}$ & Age, years & IQR & IR & $95 \% \mathrm{Cl}$ & $\mathrm{N}$ & Age, years & IQR & IR & $95 \% \mathrm{Cl}$ \\
\hline Any VHD & 34582 & 70 & $58-79$ & 75.5 & 74.7 to 76.3 & 31221 & 76 & $64-83$ & 53.0 & 52.5 to 53.6 \\
\hline Aortic stenosis & 18890 & 74 & $64-81$ & 37.8 & 37.3 to 38.3 & 17429 & 80 & $72-85$ & 24.2 & 23.8 to 24.5 \\
\hline Aortic regurgitation & 8376 & 63 & $43-73$ & 20.2 & 19.7 to 20.6 & 5477 & 71 & $57-79$ & 10.8 & 10.6 to 11.1 \\
\hline Mitral stenosis & 635 & 64 & $45-76$ & 1.5 & 1.4 to 1.6 & 1282 & 74 & $58-81$ & 2.3 & 2.2 to 2.5 \\
\hline Pulmonary stenosis & 1109 & 6 & $0-20$ & 3.7 & 3.5 to 4.0 & 1351 & 4 & $0-22$ & 4.8 & 4.5 to 5.0 \\
\hline Pulmonary regurgitation & 344 & 16 & $8-33$ & 1.0 & 0.9 to 1.2 & 293 & 19 & $8-42$ & 0.9 & 0.8 to 1.0 \\
\hline Tricuspid stenosis & 151 & 49 & $5-72$ & 0.4 & 0.4 to 0.5 & 151 & 62 & $15-76$ & 0.4 & 0.3 to 0.4 \\
\hline Tricuspid regurgitation & 1192 & 73 & $62-80$ & 2.5 & 2.3 to 2.6 & 1693 & 75 & $65-82$ & 2.8 & 2.7 to 3.0 \\
\hline Two concurrent VHD & 3656 & - & - & 8.5 & 8.2 to 8.8 & 3206 & - & - & 5.8 & 5.6 to 6.0 \\
\hline Three or more concurrent VHD & 279 & - & - & 0.7 & 0.6 to 0.8 & 291 & - & - & 0.3 & 0.3 to 0.3 \\
\hline
\end{tabular}

Incidence rate (IR) per 100000 person-years at risk between 2003 and 2010 in the Swedish population with 95\% Cls. A 3-year washout period was used between 2000 and 2002 to only include incident cases. Age is presented as median age at diagnosis and interquartile range (IQR). VHD, valvular heart disease. 

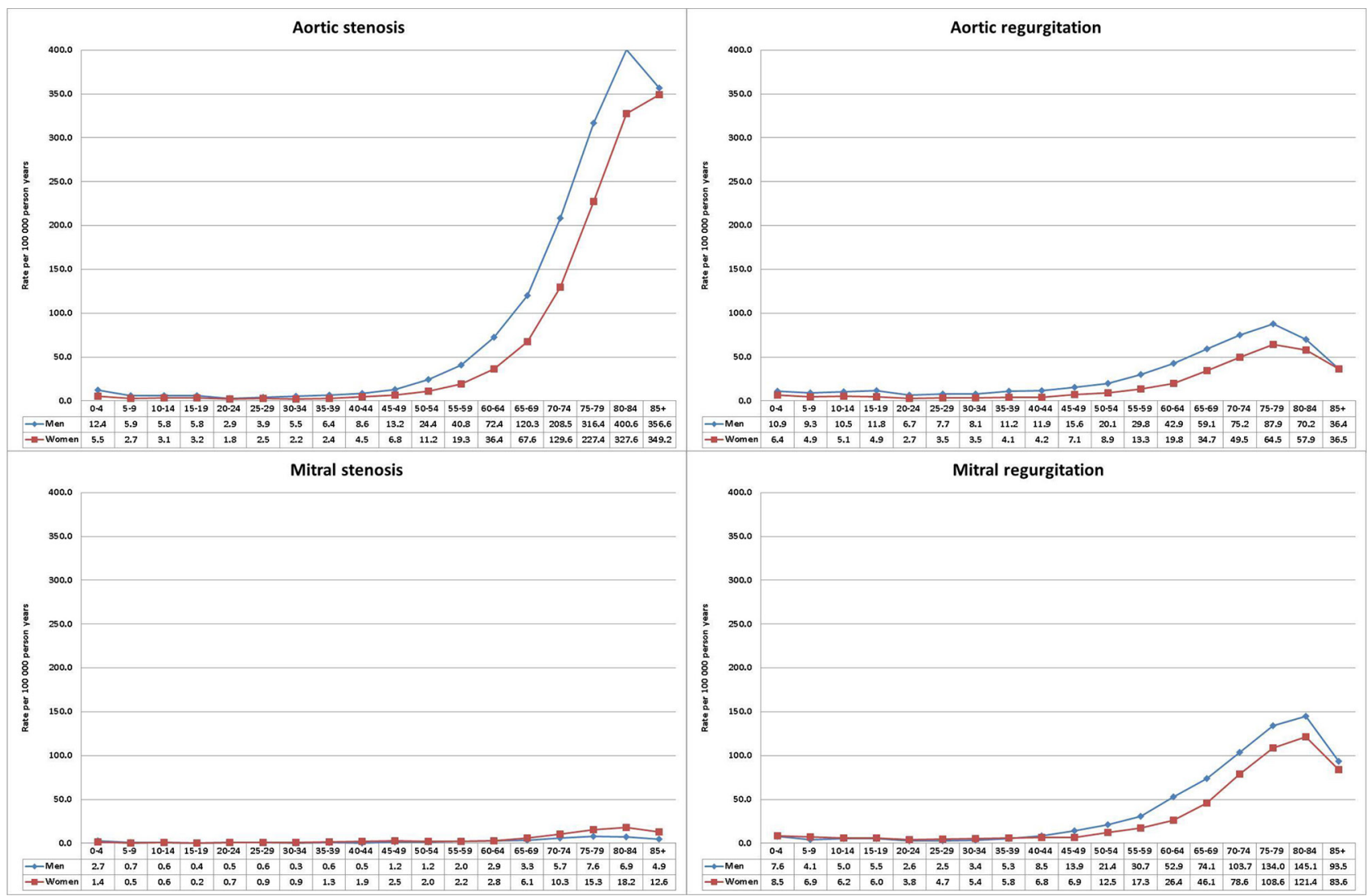

Figure 1 Incidence of left-sided valvular heart diseases in Sweden between 2003 and 2010 stratified by age and sex.
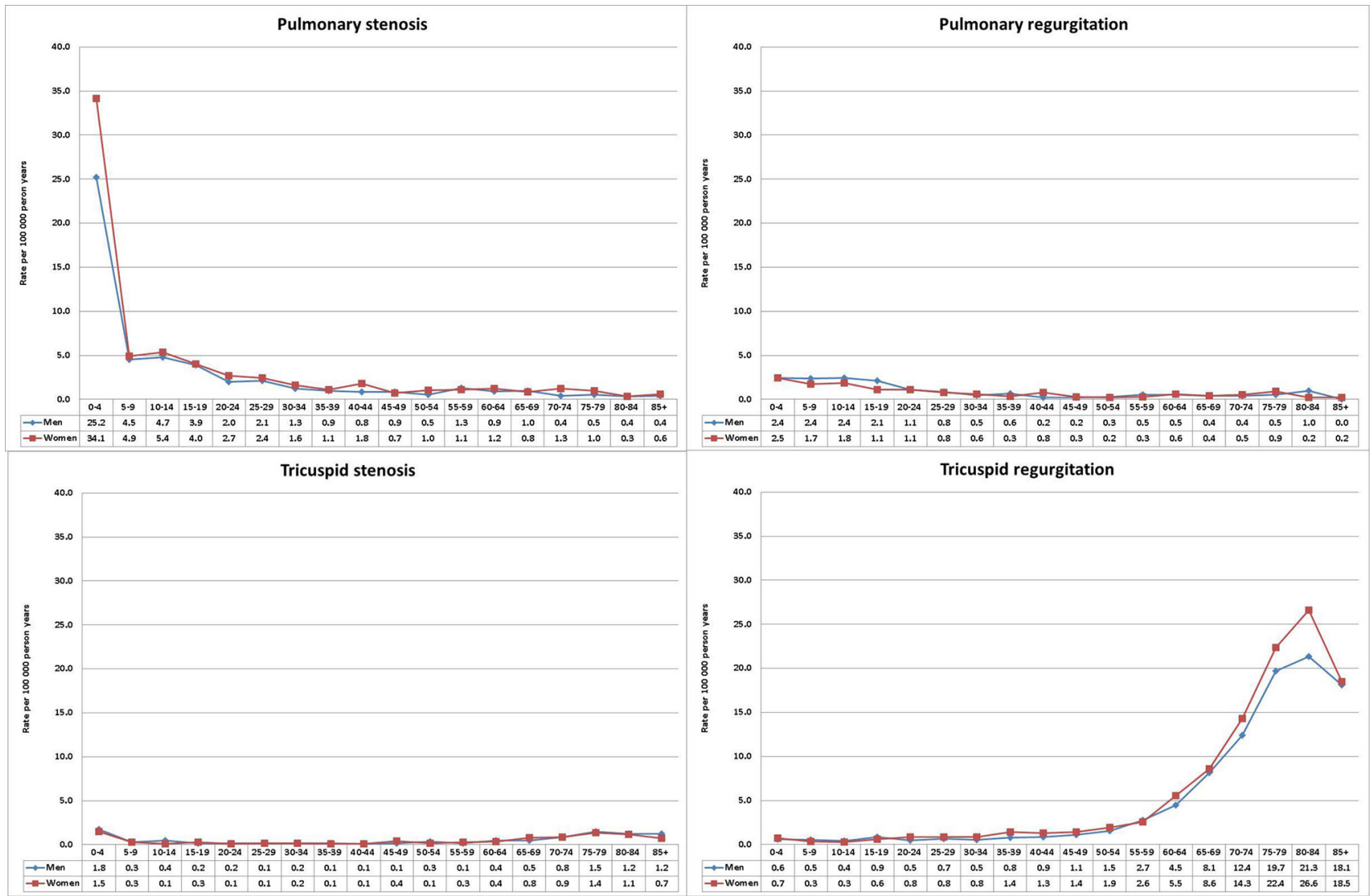

Figure 2 Incidence of right-sided valvular heart diseases in Sweden between 2003 and 2010 stratified by age and sex. 
Table 2 Concurrent combinations of valvular heart disease in Sweden between 2003 and 2010

\begin{tabular}{|c|c|c|c|c|c|c|c|c|c|c|c|c|c|c|c|c|}
\hline & \multicolumn{2}{|l|}{ AS } & \multicolumn{2}{|l|}{$A R$} & \multicolumn{2}{|l|}{ MS } & \multicolumn{2}{|l|}{ MR } & \multicolumn{2}{|l|}{ PS } & \multicolumn{2}{|l|}{ PR } & \multicolumn{2}{|l|}{ TS } & \multicolumn{2}{|l|}{ TR } \\
\hline & $\mathrm{N}$ & $\%$ & $\mathrm{~N}$ & $\%$ & $\mathrm{~N}$ & $\%$ & $\mathrm{~N}$ & $\%$ & $\mathrm{~N}$ & $\%$ & $\mathrm{~N}$ & $\%$ & $\mathrm{~N}$ & $\%$ & $N$ & $\%$ \\
\hline AS & 36319 & 100 & 2482 & 17.9 & 543 & 28.3 & 1849 & 9.9 & 54 & 2.2 & 13 & 2.0 & 37 & 12.3 & 206 & 7.1 \\
\hline AR & 2482 & 6.8 & 13853 & 100.0 & 117 & 6.1 & 1488 & 8.0 & 75 & 3.0 & 33 & 5.2 & 11 & 3.6 & 156 & 5.4 \\
\hline MS & 543 & 1.5 & 117 & 0.8 & 1917 & 100.0 & 344 & 1.8 & 8 & 0.3 & 4 & 0.6 & 12 & 4.0 & 60 & 2.1 \\
\hline MR & 1849 & 5.1 & 1488 & 10.7 & 344 & 17.9 & 18595 & 100.0 & 47 & 1.9 & 33 & 5.2 & 38 & 12.6 & 830 & 28.8 \\
\hline PS & 54 & 0.1 & 75 & 0.5 & 8 & 0.4 & 47 & 0.3 & 2460 & 100.0 & 180 & 28.3 & 30 & 9.9 & 14 & 0.5 \\
\hline PR & 13 & 0.0 & 33 & 0.2 & 4 & 0.2 & 33 & 0.2 & 180 & 7.3 & 637 & 100.0 & 6 & 2.0 & 23 & 0.8 \\
\hline TS & 37 & 0.1 & 11 & 0.1 & 12 & 0.6 & 38 & 0.2 & 30 & 1.2 & 6 & 0.9 & 302 & 100.0 & 35 & 1.2 \\
\hline TR & 206 & 0.6 & 156 & 1.1 & 60 & 3.1 & 830 & 4.5 & 14 & 0.6 & 23 & 3.6 & 35 & 11.6 & 2885 & 100.0 \\
\hline
\end{tabular}

Number of cases with different combinations of concurrent valvular heart disease diagnoses in 2003-2010 and proportion of cases with each valve disease (columns) that were concurrent with each of the other seven valve diseases (rows). AS, aortic stenosis; AR, aortic regurgitation; MS, mitral stenosis; MR, mitral regurgitation; PS, pulmonary stenosis;

PR, pulmonary regurgitation; TS, tricuspid stenosis; TR, tricuspid regurgitation.

with less prominent differences. Incidence of MS, however, showed a marked female predominance with twofold higher incidence in elderly women as compared with elderly men.

\section{Multiple VHDs}

A total of 3656 men and 3206 women (10.4\% of all VHD cases) were diagnosed with more than one VHD (table 1). The proportions with different VHD combinations are shown in table 2 . The most prominent overlaps were observed in regurgitation diseases and for MS; of patients diagnosed with PR $(n=637)$ or TR $(n=2885), 28.3 \%$ and $28.8 \%$ were also diagnosed with PS and MR, respectively. Of 13853 patients with AR, 17.9\% and $10.7 \%$ were also diagnosed with AS and MR, respectively. Of 18 595 patients with MR, 9.9\% were diagnosed with AS. Of 1917 patients with MS, 28.3\% were diagnosed with AS and 17.9\% with MR.

\section{Comorbidity profiles}

Unadjusted cross-sectional prevalence of 15 different comorbidity groups in the eight VHDs are shown in table 3. Age-adjusted and sex-adjusted ORs are shown in figure 3 and online Supplementary table $7 \mathrm{a}, \mathrm{b}$. Concomitant non-valvular congenital heart disease was associated with all VHD and most strongly with right-sided VHD where it was diagnosed in $55.9 \%$ of the patients with PS, 52.7\% among those with PR and $31.8 \%$ in those with TS. Rheumatic fever was rare overall but associated with multiple VHDs and significantly with MS (adjusted OR: $3.54,95 \%$ CI 1.35 to 9.30). Endocarditis was strongly associated with left-sided lesions, especially with AR (diagnosed in 5.4\%, adjusted OR: $21.84,95 \%$ CI 19.92 to 23.94$)$, MR (5.0\%, adjusted OR: 18.79 , 95\% CI 17.29 to 20.42 ) and TR. Nearly half of AS cases had concurrent atherosclerotic vascular disease (48.4\%, adjusted OR: 2.33, 95\% CI 2.27 to 2.39). Autoimmune diseases were common in AS and MS (28.7\% and 24.5\% as compared with $6.9 \%$ in the general population). Heritable connective tissue disorders (including Marfan syndrome, EhlersDanlos and Osteogenesis imperfecta) were rare but nonetheless associated with AR (0.6\%, adjusted OR: 6.40, 95\% CI 4.74 to $8.66)$ and MR. Abdominal hernia or prolapse was slightly more common with regurgitation defects. Carcinoids were rare but strongly associated with all VHDs, especially right-sided lesions. Pulmonary hypertension was most prevalent in TR $(3.6 \%$, adjusted OR: 9.22, 95\% CI 7.40 to 11.48). Ascending aortic aneurysms or dissections were common in AR (10.3\%, adjusted

Table 3 Comorbidity profiles for valvular heart disease in Sweden between 2003 and 2010

\begin{tabular}{|c|c|c|c|c|c|c|c|c|c|c|c|c|c|c|c|c|c|c|}
\hline \multirow[b]{2}{*}{ Total cases } & \multicolumn{2}{|l|}{ Population } & \multicolumn{2}{|l|}{ AS } & \multicolumn{2}{|l|}{ AR } & \multicolumn{2}{|l|}{ MS } & \multicolumn{2}{|l|}{ MR } & \multicolumn{2}{|l|}{ PS } & \multicolumn{2}{|l|}{ PR } & \multicolumn{2}{|l|}{ TS } & \multicolumn{2}{|l|}{ TR } \\
\hline & 10164211 & $\%$ & 36319 & $\%$ & 13853 & $\%$ & 1917 & $\%$ & 18595 & $\%$ & 2460 & $\%$ & 637 & $\%$ & 302 & $\%$ & 2885 & $\%$ \\
\hline Non-valvular congenital heart disease & 36381 & 0.4 & 800 & 2.2 & 966 & 7.0 & 120 & 6.3 & 799 & 4.3 & 1374 & 55.9 & 336 & 52.7 & 96 & 31.8 & 162 & 5.6 \\
\hline Rheumatic fever & 2847 & 0.0 & 34 & 0.1 & 21 & 0.2 & 5 & 0.3 & 24 & 0.1 & 1 & 0.0 & 0 & 0.0 & 1 & 0.3 & 2 & 0.1 \\
\hline Endocarditis & 6695 & 0.1 & 991 & 2.7 & 742 & 5.4 & 72 & 3.8 & 928 & 5.0 & 18 & 0.7 & 2 & 0.3 & 3 & 1.0 & 123 & 4.3 \\
\hline Atherosclerotic vascular disease & 616714 & 6.1 & 17572 & 48.4 & 4196 & 30.3 & 635 & 33.1 & 6755 & 36.3 & 71 & 2.9 & 40 & 6.3 & 59 & 19.5 & 970 & 33.6 \\
\hline Cancer & 670226 & 6.6 & 7235 & 19.9 & 2006 & 14.5 & 241 & 12.6 & 3386 & 18.2 & 68 & 2.8 & 26 & 4.1 & 26 & 8.6 & 577 & 20.0 \\
\hline Infections & 1948980 & 19.2 & 12634 & 34.8 & 4377 & 31.6 & 715 & 37.3 & 6479 & 34.8 & 1100 & 44.7 & 221 & 34.7 & 129 & 42.7 & 1088 & 37.7 \\
\hline Autoimmune disorders & 697905 & 6.9 & 10419 & 28.7 & 2677 & 19.3 & 469 & 24.5 & 4176 & 22.5 & 138 & 5.6 & 53 & 8.3 & 46 & 15.2 & 654 & 22.7 \\
\hline Heritable connective tissue disorders & 2785 & 0.0 & 9 & 0.0 & 81 & 0.6 & 0 & 0.0 & 45 & 0.2 & 3 & 0.1 & 1 & 0.2 & 0 & 0.0 & 4 & 0.1 \\
\hline Abdominal hernia or prolapse & 382766 & 3.8 & 3447 & 9.5 & 1324 & 9.6 & 137 & 7.1 & 1985 & 10.7 & 76 & 3.1 & 28 & 4.4 & 22 & 7.3 & 297 & 10.3 \\
\hline Carcinoid & 1323 & 0.0 & 20 & 0.1 & 12 & 0.1 & 0 & 0.0 & 22 & 0.1 & 4 & 0.2 & 4 & 0.6 & 4 & 1.3 & 31 & 1.1 \\
\hline Pulmonary hypertension & 2863 & 0.0 & 118 & 0.3 & 33 & 0.2 & 28 & 1.5 & 141 & 0.8 & 11 & 0.4 & 3 & 0.5 & 5 & 1.7 & 104 & 3.6 \\
\hline Ascending aortic aneurysm/dissection & 9846 & 0.1 & 911 & 2.5 & 1430 & 10.3 & 9 & 0.5 & 177 & 1.0 & 2 & 0.1 & 3 & 0.5 & 4 & 1.3 & 17 & 0.6 \\
\hline Atrial fibrillation & 296450 & 2.9 & 9546 & 26.3 & 3118 & 22.5 & 749 & 39.1 & 7178 & 38.6 & 66 & 2.7 & 46 & 7.2 & 81 & 26.8 & 1473 & 51.1 \\
\hline Heart failure & 237209 & 2.3 & 11646 & 32.1 & 2890 & 20.9 & 664 & 34.6 & 7301 & 39.3 & 74 & 3.0 & 37 & 5.8 & 70 & 23.2 & 1367 & 47.4 \\
\hline Non-valvular thoracic surgery & 132096 & 1.3 & 6005 & 16.5 & 2614 & 18.9 & 323 & 16.8 & 3245 & 17.5 & 409 & 16.6 & 80 & 12.6 & 103 & 34.1 & 533 & 18.5 \\
\hline
\end{tabular}

For each valvular heart disease, numbers and percentages of cases with concomitant comorbidities, defined by International Classification of Diseases codes, are shown. The numbers and percentages in the total population are also shown for comparison. AS, aortic stenosis; AR, aortic regurgitation; MS, mitral stenosis; MR, mitral regurgitation; PS, pulmonary stenosis; PR, pulmonary regurgitation; TS, tricuspid stenosis; TR, tricuspid regurgitation. 

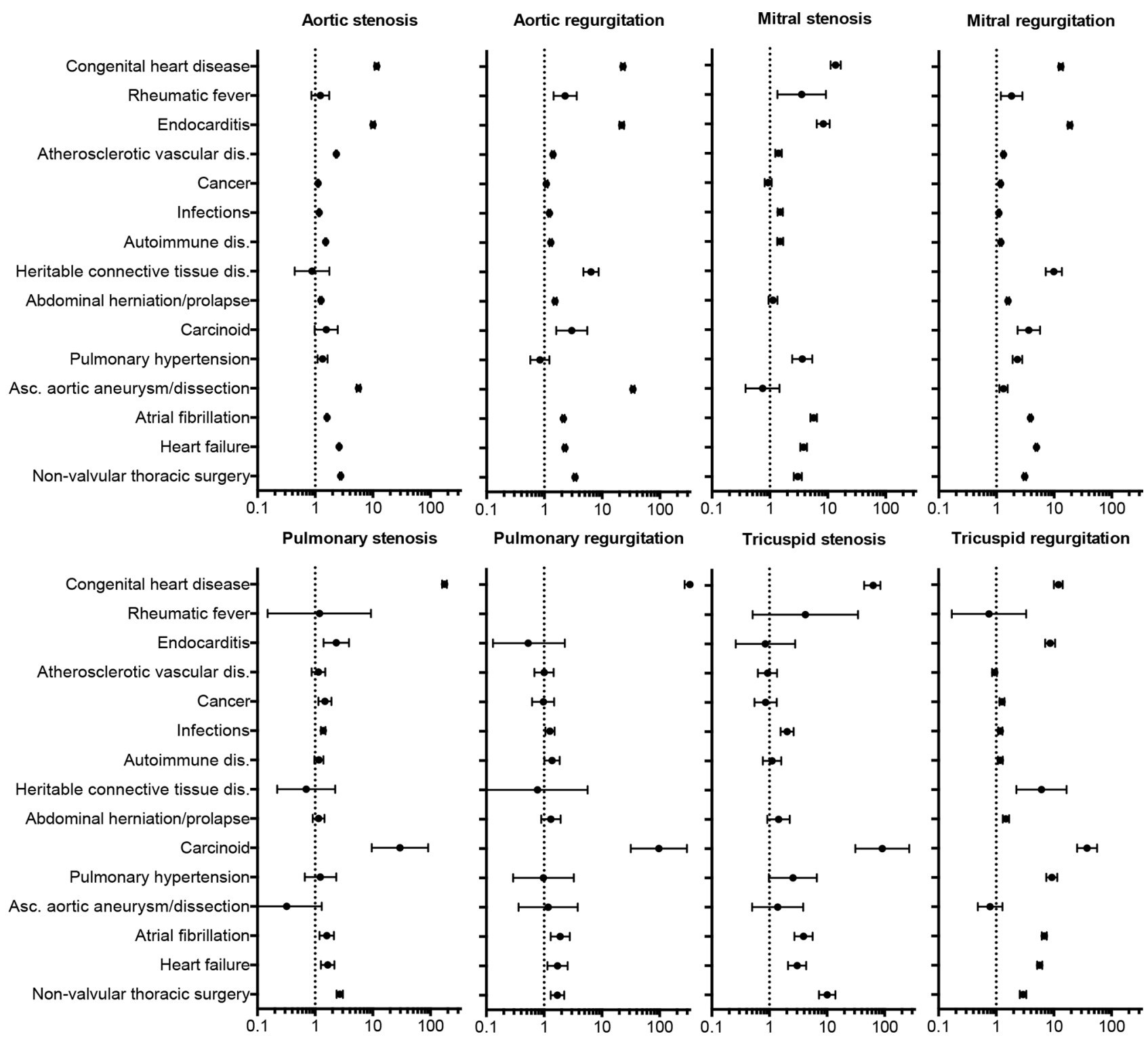

Figure 3 Comorbidity profiles for valvular heart diseases in Sweden between 2003 and 2010. The X-axis shows odds ratios on a logarithmic scale ranging from 0.1 to 300 . For each valvular heart disease, age-adjusted and sex-adjusted odds ratios for each comorbidity group with $95 \% \mathrm{Cls}$ are presented. Dis, disorders. Asc, ascending.

OR: $34.07,95 \%$ CI 31.79 to 36.53$)$. Concomitant atrial fibrillation and heart failure were most common in TR and mitral valve disease. Distributions of individual diagnoses within the most diverse comorbidity groups across VHDs are shown in the online supplementary tables $8-12$, that is, non-valvular congenital heart disease, autoimmune disorders, heritable connective tissue disorders, abdominal hernia/prolapse and non-valvular thoracic surgical procedures.

\section{DISCUSSION}

This study of the entire Swedish population underscores that VHD today remains relatively common with an overall incidence of 0.6 per 1000 person-years and that it is primarily a disease of the elderly, mostly comprised left-sided valve diseases: degenerative AS, MR and AR. These observations are in accordance with the findings of recent community echocardiographic screening studies. ${ }^{10} 27$ In addition, our large cohort allowed characterisation of the contemporary epidemiology across the whole spectrum of individual VHDs. We observed important differences between VHDs in age distribution, sex distribution and comorbidity profiles.

\section{Age distribution of individual VHDs}

Incidence of all left-sided VHDs increased continuously with age, indicating age-degenerative components. AS, in particular, is considered to represent a late manifestation of degenerative progressive calcification, with similar risk factors as atherosclerotic vascular disease. ${ }^{25-28} \mathrm{AR}$ is not strongly associated with traditional atherosclerotic risk factors but more often related to congenital abnormalities of the aortic valve leaflets or supporting structures in the aortic root and annulus, such as a bicuspid aortic valve and Marfan syndrome. ${ }^{29}{ }^{30}$ However, AR is also strongly associated with aortic aneurysms, thought to reflect ageing-related changes in the vascular wall. MR in the developed world is mainly associated with primary degenerative causes (myxomatous valvular degeneration and valve prolapse) and commonly to annular dilatation related to left ventricular dilatation or ischaemic heart disease (termed secondary or 'functional' MR). ${ }^{31}$ 
Right-sided valve diseases, however, were rare and usually presented during the first decade of life, reflecting congenital conditions. PS was the most common VHD in the young and has previously been described to be the third most frequent congenital cardiac malformation, most often resulting from incomplete separation of valvular leaflets, or as component in the tetralogy of Fallot, presenting with cardiac murmur and cyanosis resulting in early balloon valvuloplasty or surgery. ${ }^{32} \mathrm{~A}$ known complication to such interventions for PS is PR development, ${ }^{33}$ as illustrated by the incidence peak a few years after PS in this study. TS was exceedingly rare with the lowest incidence rate of any VHD. The only right-sided valve disease where the incidence showed a positive correlation with age was TR, consistent with a degenerative aetiology or a consequence of left-sided heart disease or pulmonary disease manifesting with pulmonary hypertension and right ventricular dilation.

\section{Sex distribution of individual VHDs}

We observed sex-specific differences in VHD incidence, most strikingly for AR and MS: AR was more common in men, whereas MS was more common in women. These findings are consistent with smaller case series reported previously but are not well recognised. ${ }^{34} 35$ In the developing world, rheumatic heart disease is the most common cause of both AR and MS ${ }^{30}{ }^{36}$ However, in developed countries, AR frequently occurs in combination with a congenital bicuspid valve and aortic aneurysmal dilation, whereas MS is less common and remains mainly related to rheumatic heart disease or to calcific degenerative disease. ${ }^{37}$ We have previously reported that incidence of both aneurysms in the ascending and descending aorta and aortic dissection is substantially higher in middle-aged men than women from the Swedish population and that hypertension is a major risk factor. ${ }^{38}$ We observed an early separation of sex-specific AR incidence rates, implicating a role for congenital conditions such as bicuspid aortic valves. Indeed, we also observed that the diagnosis code used to code for bicuspid aortic valves was twofold more common as cause of AS/AR in men as compared with women. However, the unclear validity and sensitivity of this code does not allow detailed assessment of the contribution of bicuspid aortic valve to the observed sex differences.

With regard to MS, a majority of Swedish women diagnosed with MS did not have a known history of rheumatic heart disease in the nationwide registers and in our validation study based on the review of patient records, consistent with low immigration to Sweden from areas with high burden of rheumatic heart disease and suggesting that age-related calcification may be the most important cause of MS today. It cannot be ruled out that MS might represent a late manifestation of undiagnosed rheumatic heart disease. Interestingly, both rheumatic $\mathrm{MS}^{3639}$ and mitral annular calcification $^{4041}$ have been described in case series to be more common in women, whereas aortic valve calcification is more common in men. Recent investigations suggest that women with AS have a more fibrotic remodelled valve indicating different pathophysiological processes. ${ }^{42}$ The mechanisms underlying these observations remain unclear, and mechanistic studies are warranted.

\section{Comorbidity profiles of individual VHDs}

We also observed distinct comorbidity profiles for different VHDs, some of which likely reflect causation (such as atherosclerotic risk factors in AS), whereas others are likely to reflect reverse causation (such as heart failure in MR) or confounding. Pulmonary valve diseases and tricuspid stenosis often coexisted with non-valvular congenital heart disease, highlighting the large extent of combined manifestations seen in congenital heart diseases. Rheumatic fever was very rare, whereas endocarditis was common in left-sided lesions and TR. Atherosclerotic vascular disease was prevalent in nearly half of all AS cases, consistent with the increasing view of AS as a late atherosclerotic manifestation. ${ }^{43}$ Autoimmune diseases were common in patients with AS or MS, for MS that may be attributable to the female predominance of both conditions. Also, rheumatic fever represents an autoimmune manifestation. As expected, heritable connective tissue disorders were rare but were strongly associated with regurgitation defects, suggesting overlapping pathophysiological pathways. Similarly, abdominal hernia and prolapse were also more common in regurgitation defects, and future studies may provide insights into potential shared structural defects. Concomitant thoracic aortic aneurysms or dissections were strikingly common in AR, consistent with shared pathophysiology. ${ }^{30}$ Concomitant heart failure and atrial fibrillation were common in mitral valve disease and TR. Primary MR may precipitate heart failure by reducing the effective stroke volume, but MR also frequently results from annular dilation secondary to left ventricular dilatation in heart failure or ischaemic heart disease. Mitral valve disease often results in atrial enlargement, providing a substrate for valvular atrial fibrillation. ${ }^{44}$ The association between TR and heart failure is less well understood, but the majority of TR cases are believed to be functional, resulting from right ventricular or atrial dilatation due to atrial fibrillation, pulmonary hypertension or left-sided heart disease.

\section{Study limitations}

The design of this study has inherent limitations that necessitate consideration. First, it is an observational study based on nationwide hospital-based healthcare registers that relies on ICD codes for inpatient and outpatient diagnoses for disease classification. Cases solely diagnosed and managed in the community setting are not included in the registers. Second, data from echocardiography were not available, although we performed validation analyses in a subset of patients and found the validity to be high. Third, a trace of valvular regurgitation can be seen on echocardiography in most individuals but may not lead to a VHD diagnosis or clinical manifestations. Therefore, the incidence rates may reflect clinically evident disease rather than true estimates of all VHD in the population. Fourth, data on the severity of VHD were not available. However, the validation analyses suggest that most diagnosed VHD cases represent moderate to severe disease, although the distribution of severity grades differed across VHDs, reflecting how cutoffs for clinical relevance are perceived differently across the heterogeneous VHD population. Finally, comorbidity groups were based on broad pathophysiological disease categories to explore potential causal mechanisms. We were therefore mostly unable to detect associations with individual diagnoses with the current strategy. Any observed associations should also be viewed as correlations and not taken as proof of causality without further evidence.

\section{CONCLUSION}

Clinically diagnosed VHD in the 21st century was common and primarily consisted of left-sided degenerative lesions in the elderly. Right-sided valve disease with pulmonary valve lesions and TS were typically diagnosed during the first decade of life. Rheumatic fever was rare in Sweden; instead specific VHDs showed different comorbidity profiles, indicating a range of potential aetiologies in the current era. AR and MS displayed striking sex-specific patterns for which the mechanisms remain incompletely understood. 


\section{Key messages}

\section{What is already known on this subject?}

Clinical case series have shown transitions in the spectrum of valvular heart disease (VHD) in the 20th century in the developed world. VHD related to rheumatic heart disease has declined, whereas VHD related to age-degenerative processes has increased.

\section{What might this study add?}

This large study of the entire Swedish population using nationwide hospital-based registers expands epidemiological understanding of VHD to individual diagnoses. The overall incidence of VHD was estimated to 63.9 per 100000 personyears, and the three most common diseases were aortic stenosis $(47.2 \%)$, mitral regurgitation (24.2\%) and aortic regurgitation (18.0\%). Specific VHDs showed different sex patterns and comorbidity profiles, indicating a range of potential aetiologies in the current era that merit further study.

\section{How might this impact on clinical practice?} Contemporary population-based epidemiological information on VHD is important to understand this changing disease spectrum, and the gained knowledge will help to allocate resources in healthcare systems more efficiently.

Acknowledgements We wish to recognise the work devoted to the development and maintenance of the national registers used in this study. We want to thank all clinicians, administrative personnel and data managers across Swedish hospitals and the Swedish National Board of Health and Welfare for making this study possible.

Contributors All authors made substantial contributions to the conception and design of the work. PA drafted the work and all authors revised it critically for important intellectual content. All authors have given final approval of the version to be published. All authors agree to be accountable for all aspects of the work in ensuring that questions related to the accuracy or integrity of any part of the work are appropriately investigated and resolved. KS and JGS jointly supervised this work.

Funding The European Research Council, Swedish Heart-Lung Foundation, the Wallenberg Center for Molecular Medicine in Lund, the Crafoord Foundation, the Swedish National Health Service, Skåne University Hospital in Lund (PA, JGS and AM) and the Swedish Research Council (XL, AM, JGS and KS).

Competing interests None declared.

Patient consent Register study, not applicable.

Ethics approval Regional Ethics committee at Lund University.

Provenance and peer review Not commissioned; externally peer reviewed.

Open Access This is an Open Access article distributed in accordance with the Creative Commons Attribution Non Commercial (CC BY-NC 4.0) license, which permits others to distribute, remix, adapt, build upon this work non-commercially, and license their derivative works on different terms, provided the original work is properly cited and the use is non-commercial. See: http://creativecommons.org/ licenses/by-nc/4.0/

(c) Article author(s) (or their employer(s) unless otherwise stated in the text of the article) 2017. All rights reserved. No commercial use is permitted unless otherwise expressly granted.

\section{REFERENCES}

1 Bland EF. Rheumatic fever: the way it was. Circulation 1987;76:1190-5

2 Cutler E, Levine S. Cardiotomy and valvulotomy for mitral stenosis. Boston Med Surg J 1923;188:1023.

3 Edler I, Lindström K. The history of echocardiography. Ultrasound Med Biol 2004;30:1565-644.

4 Rheumatic fever and rheumatic heart disease. World Health Organ Tech Rep Ser 2004;923:1-122.

5 Remenyi B, Carapetis J, Wyber R, et al; World Heart Federation. Position statement of the world heart federation on the prevention and control of rheumatic heart disease. Nat Rev Cardiol 2013;10:284-92.
6 lung B, Baron G, Butchart EG, et al. A prospective survey of patients with valvular heart disease in Europe: the euro heart survey on valvular heart disease. Eur Heart $J$ 2003;24:1231-43.

7 Nkomo VT, Gardin JM, Skelton TN, et al. Burden of valvular heart diseases: a population-based study. Lancet 2006;368:1005-11.

8 Yeh RW, Sidney S, Chandra M, et al. Population trends in the incidence and outcomes of acute myocardial infarction. N Engl J Med 2010;362:2155-65.

9 d'Arcy JL, Prendergast BD, Chambers JB, et al. Valvular heart disease: the next cardiac epidemic. Heart 2011;97:91-3.

10 d'Arcy JL, Coffey S, Loudon MA, et al. Large-scale community echocardiographic screening reveals a major burden of undiagnosed valvular heart disease in older people: the OxVALVE population cohort study. Eur Heart J 2016;37:3515-22.

11 Olsson M, Granström L, Lindblom $D$, et al. Aortic valve replacement in octogenarians with aortic stenosis: a case-control study. J Am Coll Cardiol 1992;20:1512-6.

12 Olsson M, Janfjäll H, Orth-Gomér K, et al. Quality of life in octogenarians after valve replacement due to aortic stenosis. A prospective comparison with younger patients. Eur Heart J 1996;17:583-9.

13 Shapira OM, Kelleher RM, Zelingher J, et al. Prognosis and quality of life after valve surgery in patients older than 75 years. Chest 1997;112:885-94.

14 Martinsson A, Li X, Andersson C, et al. Temporal trends in the incidence and prognosis of aortic stenosis: a nationwide study of the Swedish population. Circulation 2015;131:988-94

15 Leon MB, Smith CR, Mack M, et al; PARTNER Trial Investigators. Transcatheter aorticvalve implantation for aortic stenosis in patients who cannot undergo surgery. $N$ Engl J Med 2010;363:1597-607.

16 Smith CR, Leon MB, Mack MJ, et al; PARTNER Trial Investigators. Transcatheter versus surgical aortic-valve replacement in high-risk patients. $N$ Eng/ J Med 2011;364:2187-98.

17 Feldman T, Foster E, Glower DD, et al; EVEREST II Investigators. Percutaneous repair or surgery for mitral regurgitation. N Engl J Med 2011;364:1395-406.

18 Adams DH, Popma JJ, Reardon MJ, et al; U.S. CoreValve Clinical Investigators. Transcatheter aortic-valve replacement with a self-expanding prosthesis. $N \mathrm{Eng} / \mathrm{J}$ Med 2014;370:1790-8

19 McElhinney DB, Hellenbrand WE, Zahn EM, et al. Short- and medium-term outcomes after transcatheter pulmonary valve placement in the expanded multicenter US melody valve trial. Circulation 2010;122:507-16.

20 Schofer J, Bijuklic K, Tiburtius C, et al. First-in-human transcatheter tricuspid valve repair in a patient with severely regurgitant tricuspid valve. J Am Coll Cardiol 2015;65:1190-5

21 Ahmed MI, Aban I, Lloyd SG, et al. A randomized controlled phase Ilb trial of beta(1)receptor blockade for chronic degenerative mitral regurgitation. J Am Coll Cardiol 2012;60:833-8.

22 Bull S, Loudon M, Francis JM, et al. A prospective, double-blind, randomized controlled trial of the angiotensin-converting enzyme inhibitor ramipril in aortic stenosis (RIAS trial). Eur Heart J Cardiovasc Imaging 2015;16:834-41.

23 Singh JP, Evans JC, Levy D, et al. Prevalence and clinical determinants of mitral, tricuspid, and aortic regurgitation (the framingham heart study). Am J Cardiol 1999:83:897-902.

24 Ludvigsson JF, Andersson E, Ekbom A, et al. External review and validation of the Swedish National Inpatient Register. BMC Public Health 2011;11:450.

25 Thanassoulis G, Campbell CY, Owens DS, et al; CHARGE Extracoronary Calcium Working Group. Genetic associations with valvular calcification and aortic stenosis. N Engl J Med 2013;368:503-12.

26 Smith JG, Luk K, Schulz CA, et al; Cohorts for Heart and Aging Research in Genetic Epidemiology (CHARGE) Extracoronary Calcium Working Group. Association of lowdensity lipoprotein cholesterol-related genetic variants with aortic valve calcium and incident aortic stenosis. JAMA 2014;312:1764-71.

27 Eveborn GW, Schirmer H, Heggelund G, et al. The evolving epidemiology of valvular aortic stenosis. The Tromsø Study. Heart 2013;99:396-400.

28 Maganti K, Rigolin VH, Sarano ME, et al. Valvular heart disease: diagnosis and management. Mayo Clin Proc 2010;85:483-500.

29 Milewicz DM, Dietz HC, Miller DC. Treatment of aortic disease in patients with Marfan syndrome. Circulation 2005;111:e150-e157.

30 Enriquez-Sarano M, Tajik AJ. Clinical practice. aortic regurgitation. N Engl J Med 2004;351:1539-46.

31 Enriquez-Sarano M, Akins CW, Vahanian A. Mitral regurgitation. The Lancet 2009;373:1382-94.

32 Brickner ME, Hillis LD, Lange RA. Congenital heart disease in adults. first of two parts. N Engl J Med 2000;342:256-63.

33 Bouzas B, Kilner PJ, Gatzoulis MA. Pulmonary regurgitation: not a benign lesion. Eur Heart J 2005;26:433-9.

34 Dujardin KS, Enriquez-Sarano M, Schaff HV, et al. Mortality and morbidity of aortic regurgitation in clinical practice. A long-term follow-up study. Circulation 1999:99:1851-7.

35 Klodas E, Enriquez-Sarano M, Tajik AJ, et al. Optimizing timing of surgical correction in patients with severe aortic regurgitation: role of symptoms. J Am Coll Cardiol 1997:30:746-52

36 Chandrashekhar Y, Westaby S, Narula J, et al. Lancet 2009;374:1271-83. 
37 Carabello BA. Modern management of mitral stenosis. Circulation 2005;112:432-7.

38 Landenhed M, Engström G, Gottsäter A, et al. Risk profiles for aortic dissection and ruptured or surgically treated aneurysms: a prospective cohort study. J Am Heart Assoc 2015:4:e001513.

39 Movahed MR, Ahmadi-Kashani M, Kasravi B, et al. Increased prevalence of mitra stenosis in women. J Am Soc Echocardiogr 2006;19:911-3.

40 Kanjanauthai S, Nasir K, Katz R, et al. Relationships of mitral annular calcification to cardiovascular risk factors: the Multi-Ethnic Study of Atherosclerosis (MESA). Atherosclerosis 2010;213:558-62.
41 Elmariah S, Budoff MJ, Delaney JA, et al. Risk factors associated with the incidence and progression of mitral annulus calcification: the multi-ethnic study of atherosclerosis. Am Heart J 2013;166:904-12.

42 Simard L, Côté N, Dagenais F, et al. Sex-Related discordance between aortic valve calcification and hemodynamic severity of aortic stenosis: is valvular fibrosis the explanation? Circ Res 2017:120:681-91.

43 Carabello BA, Paulus WJ. Aortic stenosis. Lancet 2009;373:956-66.

44 De Caterina R, Camm AJ. What is 'valvular' atrial fibrillation? A reappraisal. Eur Heart J 2014;35:3328-35. 